\title{
Combined PD-1 blockade and GITR triggering induce a potent antitumor immunity in murine cancer models and synergizes with chemotherapeutic drugs
}

Lei $\mathrm{Lu}^{1+}$, Xiaobing $\mathrm{Xu}^{2+}$, Bin Zhang ${ }^{1}$, Rongsheng Zhang ${ }^{1}$, Hongzan $\mathrm{Ji}^{2^{*}}$ and Xuan Wang ${ }^{1 *}$

\begin{abstract}
Background: The coinhibitory receptor Programmed Death-1 (PD-1) inhibits effector functions of activated T cells and prevents autoimmunity, however, cancer hijack this pathway to escape from immune attack. The costimulatory receptor glucocorticoid-induced TNFR related protein (GITR) is up-regulated on activated T cells and increases their proliferation, activation and cytokine production. We hypothesize that concomitant PD-1 blockade and GITR triggering would synergistically improve the effector functions of tumor-infiltrating $T$ cells and increase the antitumor immunity. In present study, we evaluated the antitumor effects and mechanisms of combined PD-1 blockade and GITR triggering in a clinically highly relevant murine ID8 ovarian cancer model.
\end{abstract}

Methods: Mice with 7 days-established peritoneal ID8 ovarian cancer were treated intraperitoneally (i.p.) with either control, anti-PD-1, anti-GITR or anti-PD-1/GITR monoclonal antibody $(\mathrm{mAb})$ and their survival was evaluated; the phenotype and function of tumor-associated immune cells in peritoneal cavity of treated mice was analyzed by flow cytometry, and systemic antigen-specific immune response was evaluated by ELISA and cytotoxicity assay.

Results: Combined anti-PD-1/GITR mAb treatment remarkably inhibited peritoneal ID8 tumor growth with 20\% of mice tumor free 90 days after tumor challenge while treatment with either anti-PD-1 or anti-GITR mAb alone exhibited little antitumor effect. The durable antitumor effect was associated with a memory immune response and conferred by CD4 ${ }^{+}$ cells and CD8 ${ }^{+} T$ cells. The treatment of anti-PD-1/GITR mAb increased the frequencies of interferon- $\gamma$-producing effector $T$ cells and decreased immunosuppressive regulatory $T$ cells and myeloid-derived suppressor cells, shifting an immunosuppressive tumor milieu to an immunostimulatory state in peritoneal cavity. In addition, combined treatment of anti-PD-1/GITR mAb mounted an antigen-specific immune response as evidenced by antigen-specific IFN- $\gamma$ production and cytolytic activity of spleen cells from treated mice. More importantly, combined treatment of anti-PD-1/GITR mAb and chemotherapeutic drugs (cisplatin or paclitaxel) further increased the antitumor efficacy with $80 \%$ of mice obtaining tumor-free long-term survival in murine ID8 ovarian cancer and 4 T1 breast cancer models.

Conclusions: Combined anti-PD-1/GITR mAb treatment induces a potent antitumor immunity, which can be further promoted by chemotherapeutic drugs. A combined strategy of anti-PD-1/GITR mAb plus cisplatin or paclitaxel should be considered translation into clinic.

Keywords: PD-1, GITR, Monoclonal antibody, Immunotherapy

\footnotetext{
*Correspondence: jihongzan@126.com; Wangxuan_jindu@163.com

'Equal contributors

2Department of Gastroenterology and Hepatology, Jinling Hospital, Clinical School of Nanjing, Second Military Medical University, Nanjing 210002, China 'Department of Surgical Oncology, Jindu Hospital, Nanjing 210002, China
} 


\section{Background}

Epithelial ovarian carcinoma (EOC) is the most lethal gynecological tumor in women, with 22,280 new cases and 15,460 deaths estimated in the United States for 2012 [1]. Five-year overall survival is approximately 45\%, and, even with modern surgical and chemotherapeutic strategies, most cases with advanced disease relapse and succumb to the disease $[2,3]$. Thus, it is major requisite to develop novel strategies for improving the outcomes of ovarian cancer.

Emerging evidence indicates that EOC should be amenable to the immunotherapy [4]. Previous studies show that EOC cells express many tumor-associated antigens against which specific immune responses can be detected [5-9]. The pioneer studies by Coukos and colleagues further indicate immune response in tumor tissue is associated with clinical outcome of patients with EOC as evidenced by the close correlation between patient survival and tumor infiltration with $\mathrm{CD}^{+}{ }^{+} \mathrm{T}$ cells in the large annotated clinical samples [10]. In addition, in patients with EOC metastases are frequently restricted to the peritoneal cavity where the tumor is directly accessible, obviating the need for systemic delivery of immune-modulatory agents [11]. Despite the abundant evidence supporting EOC immunotherapy, clinical success with immune-based therapies for EOC has generally been modest [12].

Programmed Death 1 (PD-1) protein is a key coinhibitory receptor which is inducibly expressed on activated $\mathrm{T}$ cells, $\mathrm{B}$ cells, macrophages, dendritic cells (DC) and monocytes. PD-1 has been shown to inhibit both adaptive and innate immune response when engagement of its ligands PD-L1 (B7-H1) and PD-L2 (B7-DC), which are expressed by tumor cells, stromal cells, or both $[13,14]$. PD-L1 is the primary PD-1 ligand that is upregulated in solid tumors, where it can inhibit cytokine production and the cytolytic activity of $\mathrm{PD}-1^{+}$tumorinfiltrating $\mathrm{CD}^{+}$and $\mathrm{CD}^{+} \mathrm{T}$ cells $[15,16]$. Blockade of PD-1/PD-L1 interaction induces potent antitumor effects in animal models $[14,17,18]$; furthermore, recent clinical trials show that monoclonal antibodies (mAbs) specific for PD-1 and PD-L1 mount an impressive antitumor effect in several types of solid tumors with complete regression observed in some patients [19-21], demonstrating PD-1/ PD-L1 pathway as a highly promising target for cancer immunotherapy.

Glucocorticoid-induced TNFR related protein (GITR; a.k.a. TNFRSF18) is a co-stimulatory molecule of TNF receptor family expressed on activated T cells, B cells, NK and myeloid cells and regulatory T cells (Treg) [22]. As a co-stimulatory molecule, GITR engagement increases proliferation, activation, and cytokine production of $\mathrm{CD}^{+}$and $\mathrm{CD}^{+} \mathrm{T}$ cells [22]. GITR-specific agonistic mAbs or recombinant GITR ligand (GITRL) fusion proteins have been shown to induce tumor regression in vivo through the activation of $\mathrm{CD}^{+} \mathrm{T}$ cells, $\mathrm{CD}^{+}$ $\mathrm{T}$ cells and NK cells in several tumor models [23-25]. In addition, GITR triggering can also abrogate the immunosuppressive activity of natural Treg [26]; however, evidence indicates that the expansion of $\mathrm{CD}^{+}$effector cells, rather than Treg inhibition, is the primary mechanism underlying the antitumor effects mediated by GITRtargeting mAbs [27]. A humanized GITR-targeting mAb (TRX518) is currently being evaluated in Phase I clinical trials treating patients with late-stage melanoma [28].

Although antagonist PD-1 or agonistic GITR mAbs can promote the rejection of some murine tumors, however, poorly immunogenic tumors such as ID8 ovarian cancer do not respond to single immunomodulating mAb therapy [29]. We hypothesized that combined PD-1 blockade and GITR triggering could synergistically potentiate the antitumor immune response. In this study, using ID8 murine ovarian cancer model, we evaluated the antitumor effects and underlying mechanisms of combined anti-PD-1/GITR mAb treatment.

\section{Methods}

Mice

Female C57BL (6-8 week old) were purchased from the Model Animal Research Center of Nanjing University. Animal use was approved by the Institutional Review Board of Jindu Hospital, Nanjing, China.

\section{Cell culture}

ID8, a clone of the MOSEC ovarian carcinoma of C57BL/6 origin was a gift from Dr. George Coukos (University of Pennsylvania, Philadelphia, USA). Murine 4. T1 breast cancer cells (BALB/c background) and T cell lymphoma EL4 cells (C57BL/6 background) were kindly sent by Dr. Pu Liu (University of Washington, WA, USA). Tumor cells were cultured in the complete DMEM medium supplemented with 10\% FBS (Thermo Scientific, Rockford, IL), $100 \mathrm{U} / \mathrm{mL}$ penicillin and $100 \mu \mathrm{g} / \mathrm{mL}$ streptomycin before cell suspensions were prepared and transplanted to mice. The EL4 cells and splenocytes were maintained in a complete medium of RPMI-1640 supplemented with 10\% FBS, $25 \mathrm{mM}$ HEPES, $2 \mathrm{mM}$ glutamine, $100 \mathrm{U} / \mathrm{mL}$ penicillin and $100 \mu \mathrm{g} / \mathrm{mL}$ streptomycin.

\section{Reagents}

Therapeutic anti-GITR (Clone DTA-1; Catalog\#:BE0063), anti-PD-1 (Clone RMP1-14; Catalog\#BE0146), anti-CD4 (Clone GK1.5; Catalog\#:BE0003-1), anti-CD8 (Clone 2.43; Catalog\#:BE0061), anti-NK1.1 (Clone PK136; Catalog\#: BE0036) and control rat IgG (Clone 2A3; Catalog\#: BE0089) monoclonal antibodies (mAb) were purchased from BioXcell (West Lebanon, NH). Antibodies used for flow cytometry were purchased from Tianjing Sungene 
(eBioscience, San Diego, CA) and eBioscience (San Diego, CA). H-2Db-restricted mesothelin-derived (MESO406-414: GQKMNAQAI) or control lymphocytic choriomeningitis virus (LCMV) glycoprotein (GP)-derived (GP33-41: KAVYNFATC) epitope peptide were synthesized by GenScript (Beijing, China) and more than $95 \%$ of purity were confirmed by HPLC. Peptides were reconstituted in DMSO with final concentration of $10 \mathrm{mg} / \mathrm{mL}$.

\section{Animal experiments}

Mice (10 mice/group) were transplanted intraperitoneally (i.p.) with $5 \times 10^{6} \mathrm{ID} 8$ cells in $0.1 \mathrm{~mL}$ of PBS on day 1 . On days 8,11 and 15, mice received the i.p. injection of $250 \mu \mathrm{g}$ of control, anti-PD-1, anti-GITR or anti-PD-1/ GITR $\mathrm{mAb}$ in $250 \mu \mathrm{L}$ of PBS. For combined $\mathrm{mAb} / \mathrm{cis}-$ platin or paclitaxel therapy experiments, mice $(10 \mathrm{mice} /$ group) bearing 8 days established ID8 ovarian cancer were first pretreated with a dose of cisplatin $(10 \mathrm{mg} / \mathrm{kg}$ in $100 \mu \mathrm{L}$ PBS $)$ or paclitaxel $(10 \mathrm{mg} / \mathrm{kg}$ in $100 \mu \mathrm{L} \mathrm{PBS})$ followed by three doses of control or anti-PD-1/GITR $\mathrm{mAb}$ at the schedule described above starting on day 9 . Long-term surviving mice from combined anti-PD-1/ GITR treatment were rechallenged i.p. with $5 \times 10^{6}$ ID8 cells The mice were weighed every other day and checked for the clinical sign of swollen bellies indicative of ascites formation and for the evidence of toxicity such as respiratory distress, mobility, weight loss, diarrhea, hunched posture, and failure to eat while histopathology was conducted on major organs (i.e., liver, kidney, intestines, lungs, and colon). Mice were euthanized when they developed ascites and had a weight increase $>30 \%$ of original weight on day 1 . For combined therapy experiments in the $4 \mathrm{~T} 1$ breast cancer model, mice (5/group) were transplanted subcutaneously (s.c.) with $5 \times 10^{5} 4 \mathrm{~T} 1$ cells in $0.1 \mathrm{~mL}$ of PBS on day 1 . On days 8 , mice were intratumorally (i.t.) treated with cispaltin or paclitaxel followed by $\mathrm{mAb}$ using the dose/schedule described in ID8 model. Two perpendicular diameters of s.c. tumors were measured every other day using a caliper and tumor volumes were calculated according to the formula: $1 / 2 \times$ (length) $\times$ (width) ${ }^{2}$. Mice were sacrificed when they seemed moribund or their tumors reached $10 \mathrm{~mm}$ in diameter. The survival of mice was recorded and overall survival was calculated. For lymphocyte depleting experiments, mice were injected i.p. with $500 \mu \mathrm{g}$ of $\mathrm{mAb}$ against $\mathrm{CD} 8, \mathrm{CD} 4$, or NK1.1, 1 day before and two days after tumor challenge, followed by injection of $250 \mu \mathrm{g}$ every 5 days throughout the experiment. The efficacy of cell depletion was verified by flow cytometric analysis of lymphocyte subsets in peripheral blood (data not shown).

\section{Evaluation of tumor-associated immune cells (TAC)}

Tumor-bearing mice were euthanized 7 days after the last treatment described as in animal experiments. To obtain
TAC, $3 \mathrm{ml}$ PBS was injected into the peritoneal cavity of mice with ID8 tumors immediately after euthanasia, their belly was massaged and the fluid was removed, filtered through a $70 \mu \mathrm{M}$ cell strainer (BD Biosciences), washed and resultant peritoneal cells (including immune cells and tumor cells) were subjected to further analysis.

For flow cytometric staining, single cell suspensions of peritoneal cells were washed with FACS staining buffer and incubated with mouse Fc receptor binding inhibitor (eBioscience) for 10 minutes before staining with mAbs (eBioscience) against mouse CD45 (clone 30-F11), CD3 (clone 145-2C11), CD4 (clone GK1.5), CD8 (clone 53-6.7), CD19 (clone eBio1D3), CD11b (clone M1/70), Gr-1 (clone RB6-8C5), CD44 (clone 1 M7) and CD62L (clone MEL-14) for 30 minutes. For intracellular staining of FoxP3 (clone FJK-16 s), cells were fixed, permeabilized, and stained following the instruction of Cytofix/Cytoperm kit (BD Bioscience). For intracellular staining of IFN- $\gamma$ (clone XMG1.2), cells were restimulated in vitro with $50 \mathrm{ng} / \mathrm{ml}$ PMA and $1 \mu \mathrm{g} / \mathrm{ml}$ ionomycin for 4 hours prior to the analysis of IFN- $\gamma$ secretion in $\mathrm{CD} 4^{+}$or $\mathrm{CD}^{+}$subsets. Flow cytometry was performed using FACSCalibur (BD Biosciences) and the lymphocyte population was selected by gating CD45-positive cells. The data were analyzed using Flow Jo software (Tree Star, Ashland, OR). All flow cytometry experiments were performed at least 3 times.

\section{Evaluation of antigen-specific immune response}

Isolated splenocytes from treated mice were cultured in the presence of $10 \mu \mathrm{g} / \mathrm{mL} \mathrm{H}$-2Db-restricted mesothelinderived (MESO406-414) or LCMV-GP (GP33-41) epitope peptide for 3 days. IFN- $\gamma$ concentration in the supernatants was determined by Mouse IFN- $\gamma$ Quantikine ELISA Kit (R\&D systems).

For mesothelin-specific CTL assays, effector cells were obtained by coculturing $5 \times 10^{6}$ splenocytes with $5 \times 10^{5}$ UV-irradiated ID8 cells for 4 days. Peptide-pulsed EL4 target cells were generated by adding $10 \mu \mathrm{g} / \mathrm{ml}$ of peptide and incubating for 4 hours. CTL activity was measured using the CytoTox96 Non-Radioactive Cytotoxicity Assay kit (Promega, Madison, WI) following the manufacturer's instructions. In brief, target cells were incubated with varying numbers of effector cells for about 4 hours, and supernatants were then analyzed for lactate dehydrogenase release. The results are expressed as percent specific lysis, calculated as (Experimental release-Spontaneous release/Total release-Spontaneous release) $\times 100$.

\section{Statistics}

Results were expressed as mean \pm SEM. All statistical analyses were performed using GraphPad Prism 5. Student's $t$ test was used to compare the statistical difference between two groups and one-way ANOVA was used to compare three or more groups. Survival 
rates were analyzed using the Kaplan-Meier method and evaluated with the log-rank test with Bonferroni correction. Significant differences were accepted at $\mathrm{p}<0.05$.

\section{Results}

Combined anti-PD-1/GITR mAb treatment induced potent antitumor effects in ID8 ovarian cancer

We first assessed the antitumor effects of either single or combined anti-PD-1/GITR mAb in murine ID8 ovarian cancer, a highly clinical relevant model with ascites formation and metastases in peritoneal cavity (Figure 1A). Group of C57BL/6 mice were i.p. transplanted with $5 \times 10^{6}$ ID8 cells on day 1 and then were treated with i.p. injection of control, anti-PD-1, antiGITR or anti-PD-1/GITR mAb on day 8, 11 and 15 . Control mAb-treated mice developed ascites about 30 days after tumor challenge and had to be sacrificed. Although either single anti-PD-1 or anti-GITR mAb exhibited little antitumor effect, combined anti-PD-1/ GITR mAb treatment significantly prolonged overall survival time of mice (Figure 1B; median survival time 31.50, $34.00,33.00$ or $>75.00$ days for control, anti-PD-1, anti-GITR or anti-PD-1/GITR mAb group respectively; $\mathrm{p}<0.01$, combined $\mathrm{mAb}$ compared to single or control $\mathrm{mAb}$ ) with $20 \%$ (2 out of 10 mice) of mice remaining tumor-free (confirmed by laparotomy) 90 days after tumor challenge (Figure 1C). The weight of tumor masses from mice treated with combined $\mathrm{mAb}$ also greatly decreased compared with that from control or single mAb treated mice (Figure 1D). A repeat of the experiment gave similar results (data not shown). Long-term surviving mice from first tumor challenge (90 days after first challenge), but not naïve mice, were resistant to a subsequent rechallenge i.p. with ID8 cells (Figure 1E), indicating that combined anti-PD-1/GITR mAb treatment mounted an antitumor memory immune response in mice. Cell depleting experiments showed that tumor protection was dependent on the $\mathrm{CD}^{+}{ }^{+}$and $\mathrm{CD} 8^{+} \mathrm{T}$ cells as removal of $\mathrm{CD} 4^{+}$or $\mathrm{CD} 8^{+}$ $\mathrm{T}$ cells abrogated the antitumor effect conferred by antiPD-1/GITR mAb treatment (Figure 1F).

\section{Combined anti-PD-1/GITR mAb treatment shifted an immunosuppressive to an immunostimulatory tumor milieu}

To define the immune mechanisms of the synergistic antitumor effects of combined PD-1 blockade and GITR triggering, we analyzed the phenotype and function of tumor-associated immune cells (TAC) harvested from peritoneal cavity of treated mice 7 days after last mAb injection. Compared with control or single $\mathrm{mAb}$, combined mAb significantly increased the percentages of effector $\mathrm{CD}^{+}{ }^{+} \mathrm{FoxP}^{-} \mathrm{T}$ cells (mean value $7.70 \%, 7.78 \%$, $11.94 \%$ and $31.50 \%$ for control, anti-PD-1, anti-GITR and anti-PD-1/GITR group respectively; $\mathrm{p}<0.01$ ) and
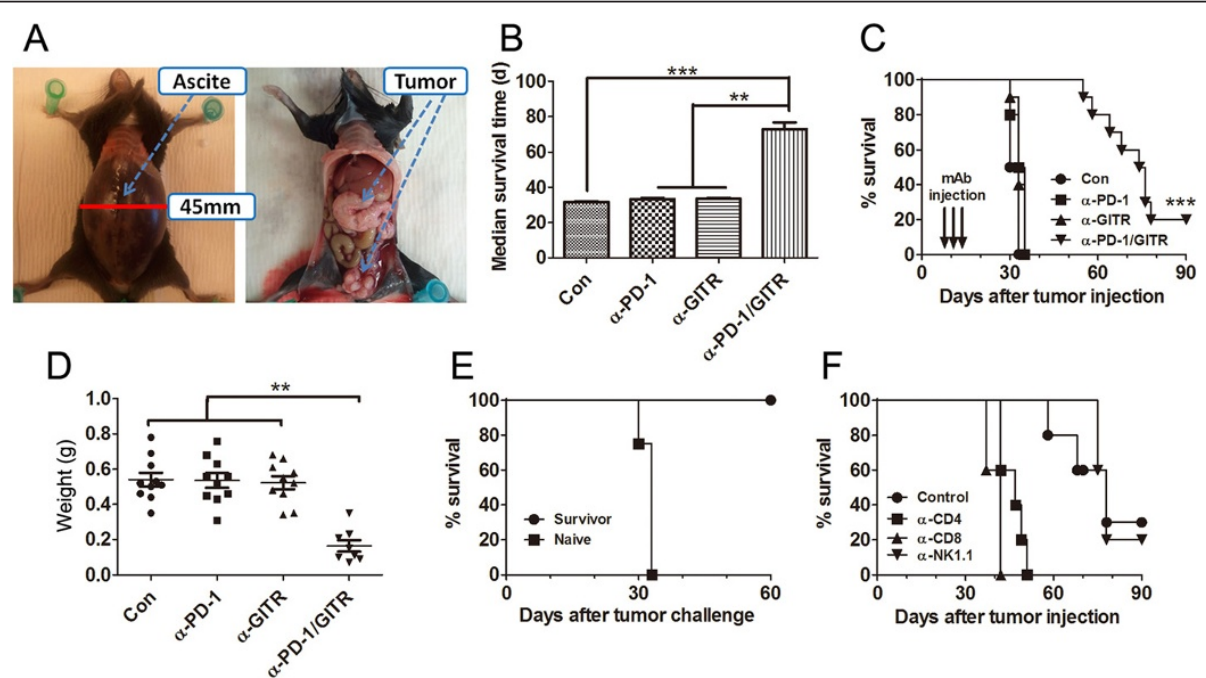

Figure 1 Treatment of combined anti-PD-1/GITR mAb induced tumor-specific long-lasting immunity against ID8 ovarian cancer. A, The typical presentation of ID8 ovarian cancer in C57BL/6 mice. The left and right picture shows the macroscopic appearance of ascites and ID8 tumor mass in peritoneal cavity of mice respectively. B. Mice (10 mice/group) were transplanted i.p. with $5 \times 10^{6}$ ID8 cells on day 1 and treated with $250 \mu \mathrm{g}$ of control, anti-PD-1, anti-GITR and anti-PD-1/GITR mAb on day 8, 11 and 15. Mean survival time of tumor-bearing mice was calculated. C, Overall survival of mice was recorded. D, The peritoneal tumor masses were weighed when mice were euthanized with each dot representing each mouse. $\mathbf{E}$, Long-term surviving (90 days after first tumor challenge) mice from combined mAb treatment group were rechallenged with ID8 cells and their overall survival was recorded. Naïve mice were challenged with ID8 cells as control. F, Mice (5 mice/group) treated with combined anti-PD-1/GITR mAb were also injected with an anti-CD4, anti-CD8, anti-NK1.1, or control mAb with $500 \mu \mathrm{g}$ of each mAb per mouse 1 day before and two days after tumor challenge followed by injection of $200 \mu \mathrm{g}$ every 5 days thereafter for the duration of the experiments and their overall survival were recorded. Data are representative of 2 independent experiments for $\mathrm{B}-\mathrm{E} .{ }^{* *} \mathrm{P}<0.05$, ${ }^{* *} \mathrm{P}<0.001$, combined $\mathrm{mAb}$ vs control or single $\mathrm{mAb}$. 
$\mathrm{CD}^{+} \mathrm{T}$ cells (mean value $11.34 \%, 12.62 \%, 17.96 \%$ and $41.04 \%$; $\mathrm{p}<0.05)$ and decreased the frequency of $\mathrm{CD}^{+}{ }^{+}$FoxP3 $^{+}$regulatory $\mathrm{T}$ cells (Treg; mean value 9.72\%, 10.44\%, 6.44\% and 2.54\%; $\mathrm{p}<0.05$ and $\mathrm{p}<0.01$ compared to anti-GITR and control or anti-PD-1 respectively) and $\mathrm{CD} 11 \mathrm{~b}^{+} \mathrm{GR}-1^{+}$myeloid-derived suppressor cells (MDSC; mean value 24.58\%, 22.28\%, 19.18\% and 8.04\%; $\mathrm{p}<0.05$ and $\mathrm{p}<0.01$ compared to anti-GITR or anti-
PD-1 and control respectively) in TAC on day 7 after treatment (Figure 2A-D); These contrasting changes in effector and immunosuppressive cells gave rise to the significantly elevated ratios of both effector $\mathrm{CD}_{4}^{+}$and $\mathrm{CD}^{+} \mathrm{T}$ cells to Treg and MDSC in peritoneal cavity of mice receiving combined $\mathrm{mAb}$ treatment (Figure $2 \mathrm{E}-\mathrm{H}$ ). With regards to individual mAb treatment, GITR engagement modestly elevated the percentage of effector

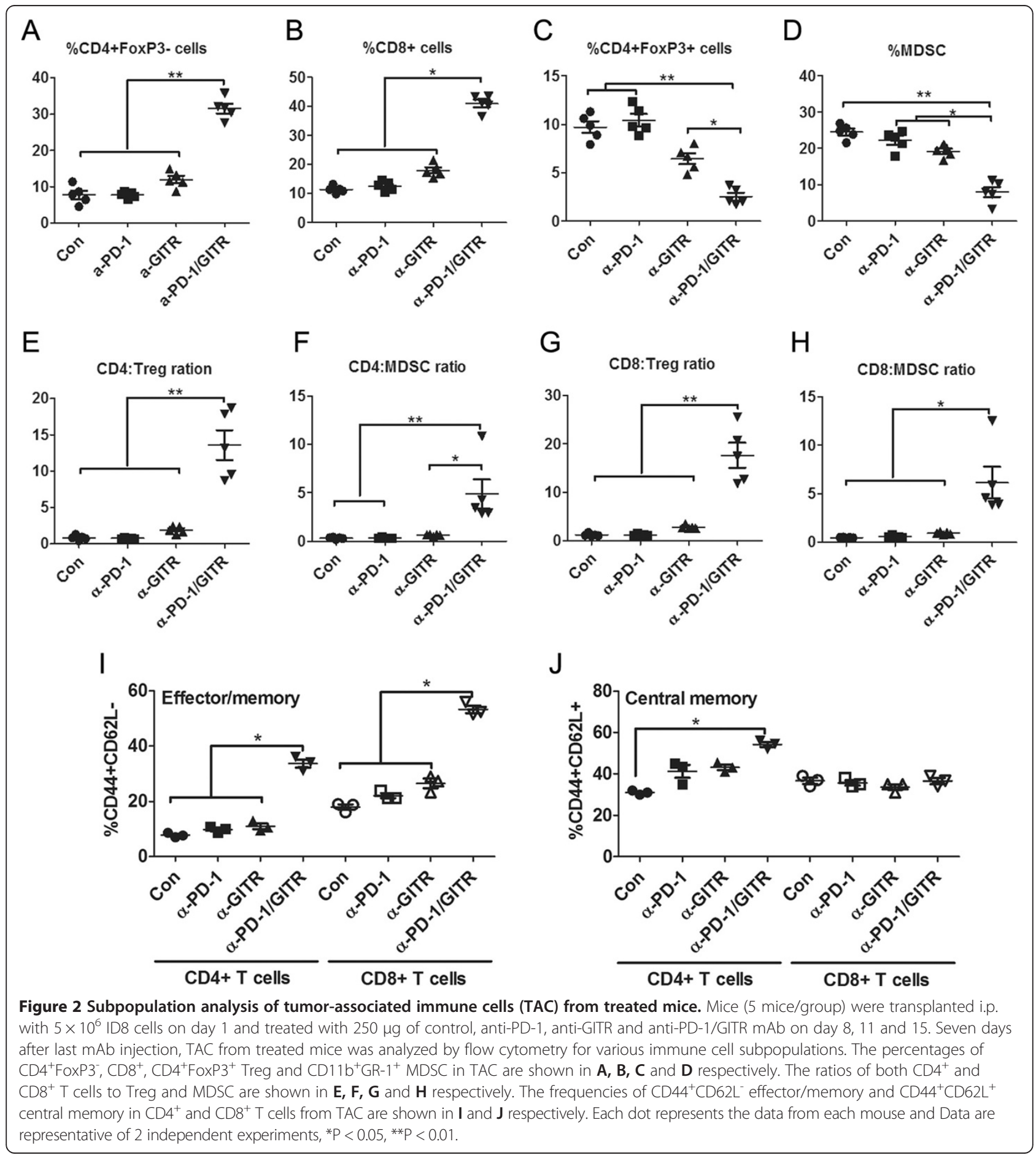


$\mathrm{CD}^{+}{ }^{+}$FoxP3 $^{-} \mathrm{T}$ cells and $\mathrm{CD} 8^{+} \mathrm{T}$ cells and attenuated the frequencies of Treg and MDSC; however, single PD-1 had little effect on these subsets. We also noted an increase in absolute number of total peritoneal immune cells from 2 mAb treated mice (mean value $\left(\times 10^{6} /\right.$ mouse): $3.8,3.9,4.2$ and 6.5 for control, anti-PD-1, anti-GITR and anti-PD-1/ GITR group respectively; $\mathrm{p}<0.05$ ) and the changes in absolute number of each subset had a similar trend to their percentages (data not shown).

Analysis of CD44 and CD62L expression on $\mathrm{CD}^{+}$and $\mathrm{CD}^{+} \mathrm{T}$ cells demonstrated that TAC from anti-PD-1/ GITR mAb treated mice contained significantly increased percentage of $\mathrm{CD} 44^{+} \mathrm{CD} 62 \mathrm{~L}^{-}$effector/memory cells (mean value $7.77 \%, 9.83 \%, 11.00 \%$ and $33.70 \%$ for CD4 cells; $17.93 \%, 22.10 \%, 26.50 \%$ and $53.30 \%$ for CD8 cells; $\mathrm{p}<0.05$ for both cells) and CD44 ${ }^{+} \mathrm{CD}_{2} 2 \mathrm{~L}^{+}$central memory cells $(31.00 \%, 41.20 \%, 43.13 \%$ and $54.17 \%$ for CD4 cells; $36.67 \%$, 35.67\%, 33.67\% and $36.57 \%$ for CD8 cells; $\mathrm{p}<0.05$ for CD4 cells) compared with that from control or single mAb treated mice (Figure 3I, J), where we could see more effector/memory cells or central memory cells in respective $\mathrm{CD}^{+}$or $\mathrm{CD}^{+}{ }^{+} \mathrm{T}$ cells from combination treatment.

Further functional analysis showed that significantly elevated frequencies of IFN- $\gamma$-producing cells were seen in tumor-associated $\mathrm{CD}^{+}$and $\mathrm{CD}^{+} \mathrm{T}$ cells from combined $\mathrm{mAb}$ treated mice (Figure $3 \mathrm{~A}$ ). The representative dotplots were shown in Figure $3 \mathrm{~B}$.

Together, the data indicate that PD-1 blockade and GITR triggering synergistically creates higher ratios of effector T cells to immunosuppressive cells in peritoneal cavity of treated mice, which represents the shift of an immunosuppressive tumor milieu to an immunostimulatory state which is more permissive for immune mediated tumor destruction.

\section{Combined anti-PD-1/GITR mAb treatment mounted an antigen-specific $C T L$ response}

We next evaluated the antigen-specific immune response in treated mice. As ID8 cancer cells express the mesothelin, a well-known tumor antigen [29,30], we harvested splenocytes from treated mice, and cultured them in the presence of $10 \mu \mathrm{g} / \mathrm{mL}$ of $\mathrm{H}$-2Db-restricted mesothelinderived epitope peptide (MESO406-414) or control GP33-41 epitope peptide for 3 days and assayed IFN- $\gamma$ secretion in culture supernatants by ELISA. As shown in Figure 4A, splenocytes from combined mAb-treated mice produced significantly higher levels of IFN- $\gamma$ when stimulated by the mesothelin epitope peptide compared to control or single mAb-treated mice $(\mathrm{P}<0.01)$. No IFN- $\gamma$ secretion were seen in the culture supernatants of splenocytes from treated mice upon stimulation by control GP33-41 epitope peptide, suggesting the elicitation of mesothelin-specific immune response in combined $\mathrm{mAb}$ treated mice. We further evaluated the antigen-specific killing activity by splenocytes from treated mice. Splenocytes were restimulated with UV-irradiated ID8 cells for 5 days before CTL assays were performed using EL4 cells pulsed with MESO406-414 or GP33-41 epitope peptide as target cells. As shown in Figure $4 \mathrm{~B}$ and $4 \mathrm{C}$, splenocytes from anti-PD-1/GITR treated mice, but not control or single mAb treated mice, exhibited a prominent cytotoxicity against EL4 cell pulsed with MESO406-414 but not GP33-41 epitope peptide, suggesting the induction of mesothelin-specific CTL response in combined $\mathrm{mAb}$ treated mice.

\section{Combined treatment of anti-PD-1/GITR mAb and chemotherapeutic drugs induced a durable antitumor effect} To mimic clinical application more closely, we evaluated whether anti-PD-1/GITR mAb could synergize with cisplatin and paclitaxel, two commonly used chemotherapeutic drugs for advanced EOC, to produce durable antitumor effects. We treated ID8 tumor-bearing mice with i.p. injection of a dose of cisplatin or paclitaxel 1 day before three doses of anti-PD-1/GITR mAb within a week (Figure 5A). Similar to the result in Figure $1 \mathrm{C}$, combined anti-PD-1/GITR mAb treatment significantly prolonged the survival of mice with $20 \%$ of mice remaining tumor-free 90 days after tumor challenge, and cisplatin or paclitaxel pretreatment alone also modestly increased the survival of mice; strikingly, combined treatment of anti-PD-1/GITR mAb plus cisplatin or paclitaxel produced an impressing antitumor effect, resulting in the long-term survival of more than $80 \%$ mice ( 8 or 9 mice out of 10 for anti-PD-1/GITR/cisplatin or anti-PD-1/ GITR/paclitaxel respectively) at the terminate of experiments (Figure 5B). With regard to treatments combining single $\mathrm{mAb}$ with chemotherapeutic drug (anti-PD-1/ cisplatin, anti-PD-1/paclitaxel, anti-GITR/cisplatin, or antiGITR/paclitaxel), we observed a similar antitumor effect as treatment with combined anti-PD-1/GITR mAb alone (data not shown).

To validate the above results, we repeated an experiment with the $4 \mathrm{~T} 1$ breast cancer model. BALB/c mice with s.c. established $4 . \mathrm{T} 1$ tumors (4-6 $\mathrm{mm}$ in diameter) were injected i.t. with anti-PD-1/GITR mAb, cisplatin, paclitaxel or a combination of $\mathrm{mAb}$ and cisplatin or paclitaxel, using the same dose/schedule as in the ID8 experiment. As shown in Figure $5 \mathrm{~B}$ and $5 \mathrm{C}$, combined anti-PD-1/GITR mAb exhibited a similar antitumor effect in this model with evident prolongation of overall survival (median survival 43 vs 25 days, $\mathrm{p}<0.05$ compared with control), however, all mice succumbed to tumor growth and died by 60 days after tumor challenge. A dose of cisplatin or paclitaxel alone exhibited a mild antitumor effect (median survival 29 or 31 vs 25 days). In contrast, a combination of anti-PD-1/GITR mAb with cisplatin or 


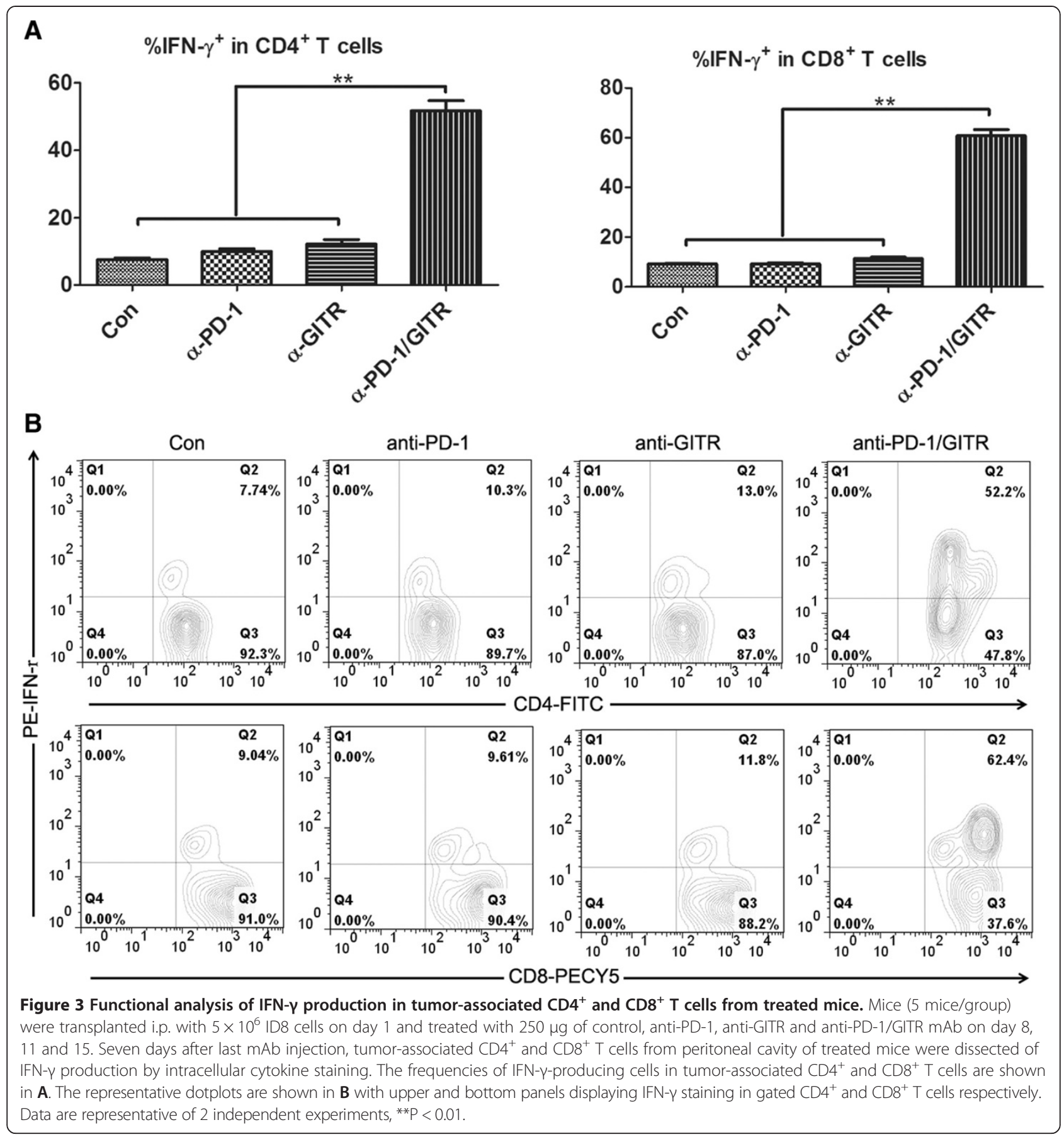

paclitaxel significantly suppressed tumor growth and $80 \%$ of mice (4 mice out of 5) survived tumor-free when the experiment was terminated 90 days after tumor challenge. These long-term surviving mice developed a systemic immune response with memory as demonstrated by their resistance to rechallenge with $4 \mathrm{~T} 1$ cells (data not shown).

\section{Discussion}

The antitumor effect of immunotherapy remains insufficient to achieve long-lasting clinical responses in patients with advanced EOC. In this study, we show that combined anti-PD-1/GITR mAb elicited a potent antitumor immune response resulting in significant tumor growth suppression in a highly clinically relevant ovarian cancer model; more importantly, anti-PD-1/GITR mAb show a clearly synergistic antitumor effect with cisplatin and paclitaxel, two most commonly used chemotherapeutic drugs for EOC patients, in murine ID8 ovarian cancer (C57BL/6 origin) and 4 T1 breast cancer (BALB/c origin) models with two strains of mice with different genetic backgrounds. Our 

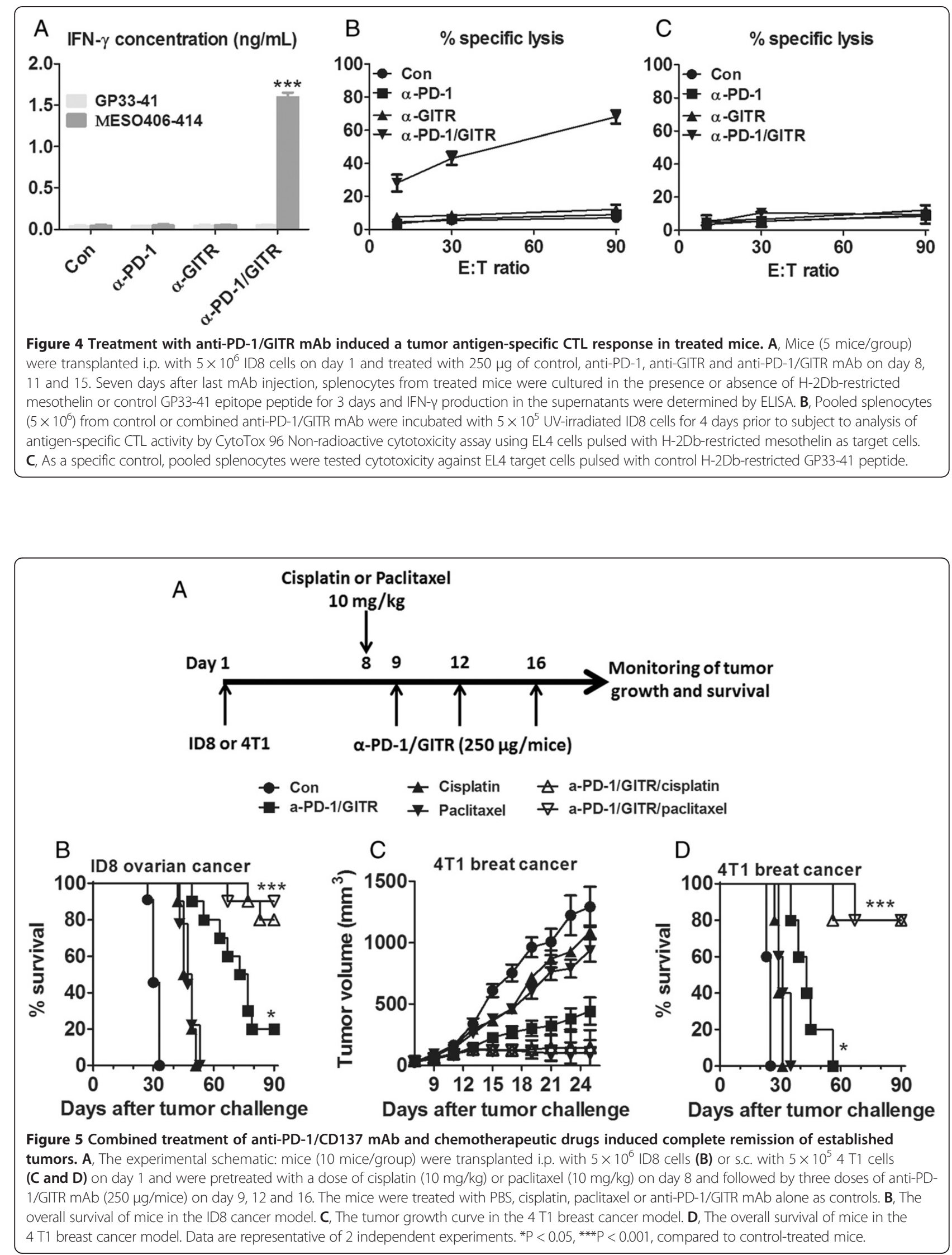
findings provide a strong rationale for translation of this combination strategy to clinic.

We defined the immune mechanisms of the therapeutic effects by combined anti-PD-1/GITR mAb. Analyzing the components of TAC from treated mice, we found that single GITR triggering slightly increased or decreased the percentages of $\mathrm{CD} 4^{+}$and $\mathrm{CD}^{+}{ }^{+} \mathrm{T}$ cells or Treg and MDSC respectively in this tumor model, which is consistent with previous reporting effects of GITR triggering on these subsets [31,32]; although single PD-1 blockade had little effect on the components of TAC, combining PD-1 blockade with GITR activation significantly promoted the accumulation of $\mathrm{CD}^{+}$and $\mathrm{CD} 8^{+} \mathrm{T}$ cells with concomitant attenuation of Treg and MDSC, giving rise to a favorable ratio of the effector $\mathrm{T}$ cells to the immunosuppressive cells which is closely correlated with the effective immunotherapy as previously stated $[33,34]$. Furthermore, increased frequencies of $\mathrm{CD}_{4} 4^{+} \mathrm{CD} 62 \mathrm{~L}^{-}$ effector/memory and/or $\mathrm{CD} 44^{+} \mathrm{CD}_{2} 2 \mathrm{~L}^{+}$central memory cells were present in $\mathrm{CD}_{4}^{+}$and $\mathrm{CD}^{+}{ }^{+} \mathrm{T}$ cells from combined $\mathrm{mAb}$ treated mice and these effector or memory $\mathrm{T}$ cells produced much more IFN- $\gamma$ in response to TCR engagement. Consistent with these observations, $\mathrm{CD} 4^{+}$ and $\mathrm{CD} 8^{+} \mathrm{T}$ cells were indispensable for tumor protection conferred by combined treatment as shown in cell depletion experiments and long-lasting memory immune response was developed as evidence by the resistance to tumor rechallenge. The data support that combined PD-1 blockade and GITR triggering shifted an immunosuppressive tumor environment to an immunostimulatory state, which favorably contributes to a durable antitumor effect.

We detected a systemic antigen-specific CTL response to ID8-expressing mesothelin in anti-PD-1/GITR mAb treated mice, as evidenced by mesothelin-specific IFN- $\gamma$ production and cytolysis by $\mathrm{CD}^{+} \mathrm{T}$ cells from these mice. We also observed an increased percentage of splenic $\mathrm{CD}^{+} \mathrm{T}$ cells in combined mAb-treated mice compared with that in control or single mAb-treated mice $(9.3 \pm 3.1 \%, 10.3 \pm 2.9 \%, 10.6 \pm 3.0 \%$ or $13.1 \pm 2.6 \%$ for control, anti-PD-1 or anti-OX40 or anti-PD-1/OX40 group); after normalization to the percentage of splenic $\mathrm{CD}^{+} \mathrm{T}$ cells, a significantly increased mesothelinspecific IFN- $\gamma$ production from combined mAb-treated mice was still seen (data not shown). As an endogenous non-mutated antigen, mesothelin should be naturally tolerized against; therefore, the induction of mesothelinspecific CTL response by anti-PD-1/GITR mAb treatment indicates that endogenous tolerance to mesothelin was broken, which is consistent with previous studies demonstrating the overcome of tolerance/ignorance by GITR activation in murine tumors and the presence of mesothelin-specific immune response in patients with cancers expressing high level of mesothelin $[24,35,36]$. We did not detect mesothelin-specific antibodies in sera from mice treated with combined mAb (data not shown).

Currently, it remains unclear for the mechanisms underlying the synergy between anti-PD-1 and antiGITR mAb. The insensitivity of ID8 ovarian cancer to treatment of single anti-PD-1 or anti-GITR mAb is in part due to lack of expression of PD-1 ligands PD-L1/2 or GITR ligand GITRL since ligand expression on the tumor has been reported to be correlated with the response to these mAbs $[19,37]$. We checked the PL-L1 expression on ID8 tumor cells after in vivo treatment by anti-GITR mAb and found de novo PL-L1 expression on these tumor cells (Additional file 1: Figure S1), which may be partially responsible for the resistance of ID8 tumors to treatment with single anti-GITR mAb and provide a rationale for the synergistic effect of PD-1 blockade and GITR activation in ID8 tumor inhibition. In addition, GITR triggering may attenuate the Tregmediated suppression of antitumor immunity [32] while PD-1 blockade can release the brake of negative signaling on effector functions of preexisting tumor-associated $\mathrm{T}$ cells. By incorporating those two stings of power, combined treatment of anti-PD-1/GITR mAb can mount a potent antitumor immunity. Clearly, more studies are warranted to define further the synergistic mechanisms in this scenario.

Importantly, addition to the anti-PD-1/GITR mAb combination of cisplatin or paclitaxel, two commonly used chemotherapeutic drugs for EOC treatment [38], administered at a dose equivalent to those used clinically [39], provided long term remission in more than $80 \%$ of the treated mice. This therapeutic effect was confirmed in the murine $4 \mathrm{~T} 1$ breast tumor model with long-term survival 4 of 5 mice receiving combined $\mathrm{mAb} /$ cisplatin or paclitaxel treatment. Interestingly, combination of either anti-PD-1 or anti-GITR mAb and chemotherapeutic drugs did not eradicate most of tumor, underscoring the necessity of synergistic PD-1 blockade and GITR triggering in eliciting an optimal antitumor effects. The detailed mechanisms of synergy between cisplatin/paclitaxel and combined immunotherapy remain unclear; however, increasing tumor antigenicity and/or further deleting immunosuppressive cells by chemotherapeutic drugs may be at least partially responsible for their synergism [40]. Future work is needed to elucidate the exact mechanism of action in this context.

With regard to GITR activation, a clinical grade of anti-GITR agonistic mAb is being tested in a phase I clinical trial [28], and GITRL fusion proteins have also shown a promising antitumor potential in preclinical tumor models [25]. In the aspect of blocking the inhibitory PD-1 pathway, PD-1- or PD-L1-targeting antagonistic mAbs have displayed an impressive antitumor effect for the treatment of advanced solid tumors with manageable 
autoimmune adverse effects $[19,21]$. It is noteworthy that consequential peritoneal administration of chemotherapeutic drugs and mAbs, a procedure that can be applied clinically in patients with ovarian cancer, did not induce any obvious toxicity such as weight or hair loss in this study. Therefore, our findings provide a strong rationale for clinical trials testing chemo-immunotherapy of ovarian cancer by combined PD-1 blockade using PD-1- or PD-L1-targeting mAb and GITR activation using GITR-specific mAb or GITRL fusion protein and chemotherapeutic drugs.

\section{Conclusions}

To our knowledge, this is the first study showing that combinatorial PD-1 blockade by an antagonistic mAb and GITR triggering by an agonistic mAb induce a potent antitumor effect, which further synergizes with commonly used chemotherapeutic drugs in two murine tumor models. Our demonstration of a synergistic antitumor effect of combining anti-PD-1/GITR mAbs with chemotherapeutic drugs should stimulate further studies to assess the safety and efficacy of similar combinatorial strategies towards 'translation' to the clinic.

\section{Additional file}

Additional file 1: Figure S1. PD-L1 expression on ex vivo ID8 tumor cells. A, PD-L1 expression on in vitro culture ID8 tumor cells was analyzed by flow cytometry using PE conjugated anti-PD-L1 (clone MIH5) or isotype control (clone eBR2a; rat lgG2a) mAb (all from eBioscience). Filled gray line indicates isotype control staining, and red line indicates PL-L1 staining. B, B6 mice were injected i.p. with $5 \times 10^{6}$ ID 8 cells and treated with $250 \mu \mathrm{g}$ of control or anti-GITR mAb on day 8. Two days later, tumor cells were collected and PD-L1 expression was analyzed by flow cytometry as above. Filled gray line indicates isotype control staining, blue line indicates tumors from control-treated mice, and green line indicates tumors from anti-GITR-treated mice.

\section{Abbreviations}

mAb: Monoclonal antibody; TNFR: Tumor necrosis factor receptor; CTL: Cytotoxicity T lymphocytes; Treg: Regulatory T cells; MDSC: Myeloid-derived suppressor cells; EOC: Epithelial ovarian carcinoma; IFN-ү: Interferon-gamma; PD-1: Programmed death protein 1; GITR: Glucocorticoid-induced TNFR related protein; DMEM: Dulbecco's minimum essential medium; RPMl: Roswell Park Memorial Institute; NK: Natural killer; DC: Dendritic cells; PBS: Phosphate-buffered saline; FBS: Fetal bovine serum; RT-PCR: Reverse transcription polymerase chain reaction; GAPDH: Glyceraldehyde phosphate dehydrogenase; SEM: Standard error of mean.

\section{Competing interests}

The authors declare that they have no competing interests.

\section{Authors' contributions}

$L L$ performed most of the experiments and drafted the manuscript. XBX carried out the flow cytometric analysis, participated in the design of the study and helped in writing the manuscript. BZ contributed in cell culture techniques and analyzed data. RSZ participated in the statistical analysis and interpretation of data. XW and HZJ conceived and designed the study and critically revised the manuscript. All authors read and approved the final manuscript.

\section{Acknowledgements}

A special thanks to Linxiong Wang for his invaluable assistance in flow cytometric analysis. This work was supported by the Nanjing City medical technology development project (No. 000 K254).

Received: 9 December 2013 Accepted: 4 February 2014 Published: 7 February 2014

\section{References}

1. Siegel R, DeSantis C, Virgo K, Stein K, Mariotto A, Smith T, Cooper D, Gansler T, Lerro C, Fedewa S, et al: Cancer treatment and survivorship statistics, 2012. CA Cancer J Clin 2012, 62(4):220-241.

2. Bast RC Jr, Hennessy B, Mills GB: The biology of ovarian cancer: new opportunities for translation. Nat Rev Cancer 2009, 9(6):415-428.

3. Jemal A, Bray F, Center MM, Ferlay J, Ward E, Forman D: Global cancer statistics. CA Cancer J Clin 2011, 61(2):69-90.

4. Kandalaft LE, Powell DJ Jr, Singh N, Coukos G: Immunotherapy for ovarian cancer: what's next? J Clin Oncol 2011, 29(7):925-933.

5. Ramakrishna V, Ross MM, Petersson M, Gatlin CC, Lyons CE, Miller CL, Myers $H E$, McDaniel M, Karns LR, Kiessling R, et al: Naturally occurring peptides associated with HLA-A2 in ovarian cancer cell lines identified by mass spectrometry are targets of HLA-A2-restricted cytotoxic T cells. Int Immunol 2003, 15(6):751-763.

6. Santin AD, Hermonat PL, Ravaggi A, Bellone S, Roman JJ, Smith CV, Pecorelli S, Radominska-Pandya A, Cannon MJ, Parham GP: Phenotypic and functional analysis of tumor-infiltrating lymphocytes compared with tumor-associated lymphocytes from ascitic fluid and peripheral blood lymphocytes in patients with advanced ovarian cancer. Gynecol Obstet Invest 2001, 51(4):254-261.

7. Schondorf T, Engel H, Kurbacher CM, Brenne U, Kolhagen H, Gohring UJ, Scharl A, Mallmann P: Immunologic features of tumor-infiltrating lymphocytes and peripheral blood lymphocytes in ovarian cancer patients. J Soc Gynecol Investig 1998, 5(2):102-107.

8. Wei H, Liu P, Swisher E, Yip YY, Tse JH, Agnew K, Hellstrom KE, Hellstrom I: Silencing of the TGF-beta1 gene increases the immunogenicity of cells from human ovarian carcinoma. J Immunother 2012, 35(3):267-275.

9. Vermeij R, Leffers N, Melief CJ, Daemen T, Nijman HW: Antigen-specific immunotherapy in ovarian cancer and p53 as tumor antigen. Curr Pharm Des 2012, 18(25):3804-3811.

10. Zhang L, Conejo-Garcia JR, Katsaros D, Gimotty PA, Massobrio M, Regnani G, Makrigiannakis A, Gray H, Schlienger K, Liebman MN, et al: Intratumoral T cells, recurrence, and survival in epithelial ovarian cancer. N Engl J Med 2003, 348(3):203-213.

11. Cubillos-Ruiz JR, Engle $X$, Scarlett UK, Martinez D, Barber A, Elgueta R, Wang $L$, Nesbeth $Y$, Durant $Y$, Gewirtz AT, et al: Polyethylenimine-based siRNA nanocomplexes reprogram tumor-associated dendritic cells via TLR5 to elicit therapeutic antitumor immunity. J Clin Invest 2009, 119(8):2231-2244.

12. Mantia-Smaldone GM, Corr B, Chu CS: Immunotherapy in ovarian cancer. Hum Vaccin Immunother 2012, 8(9):1179-1191.

13. Dong H, Zhu G, Tamada K, Chen L: B7-H1, a third member of the B7 family, co-stimulates T-cell proliferation and interleukin-10 secretion. Nat Med 1999, 5(12):1365-1369.

14. Dong H, Strome SE, Salomao DR, Tamura H, Hirano F, Flies DB, Roche PC, Lu J, Zhu G, Tamada K, et al: Tumor-associated B7-H1 promotes T-cell apoptosis: a potential mechanism of immune evasion. Nat Med 2002, 8(8):793-800.

15. Taube JM, Anders RA, Young GD, Xu H, Sharma R, McMiller TL, Chen S, Klein AP, Pardoll DM, Topalian SL, et al: Colocalization of inflammatory response with B7-h1 expression in human melanocytic lesions supports an adaptive resistance mechanism of immune escape. Sci Trans/ Med 2012, 4(127):127ra137.

16. Pardoll D, Drake C: Immunotherapy earns its spot in the ranks of cancer therapy. J Exp Med 2012, 209(2):201-209.

17. Iwai $Y$, Ishida M, Tanaka Y, Okazaki T, Honjo T, Minato N: Involvement of PD-L1 on tumor cells in the escape from host immune system and tumor immunotherapy by PD-L1 blockade. Proc Natl Acad Sci USA 2002, 99(19):12293-12297.

18. Xiao H, Huang B, Yuan Y, Li D, Han LF, Liu Y, Gong W, Wu FH, Zhang GM, Feng ZH: Soluble PD-1 facilitates 4-1BBL-triggered antitumor immunity against murine $\mathrm{H} 22$ hepatocarcinoma in vivo. Clin Cancer Res 2007, 13(6):1823-1830. 
19. Brahmer JR, Tykodi SS, Chow LQ, Hwu WJ, Topalian SL, Hwu P, Drake CG Camacho LH, Kauh J, Odunsi K, et al: Safety and activity of anti-PD-L1 antibody in patients with advanced cancer. N Engl J Med 2012, 366(26):2455-2465.

20. Topalian SL, Hodi FS, Brahmer JR, Gettinger SN, Smith DC, McDermott DF, Powderly JD, Carvajal RD, Sosman JA, Atkins MB, et al: Safety, activity, and immune correlates of anti-PD-1 antibody in cancer. N Engl J Med 2012, 366(26):2443-2454.

21. Lipson EJ, Sharfman WH, Drake CG, Wollner I, Taube JM, Anders RA, Xu H, Yao S, Pons A, Chen L, et al: Durable cancer regression off-treatment and effective reinduction therapy with an anti-PD-1 antibody. Clin Cancer Res 2013, 19(2):462-468

22. Watts TH: TNF/TNFR family members in costimulation of T cell responses. Annu Rev Immunol 2005, 23:23-68.

23. Zhou P, L'Italien L, Hodges D, Schebye XM: Pivotal roles of CD4+ effector T cells in mediating agonistic anti-GITR mAb-induced-immune activation and tumor immunity in CT26 tumors. J Immunol 2007, 179(11):7365-7375.

24. Ramirez-Montagut T, Chow A, Hirschhorn-Cymerman D, Terwey TH, Kochman AA, Lu S, Miles RC, Sakaguchi S, Houghton AN, van den Brink MR: Glucocorticoid-induced TNF receptor family related gene activation overcomes tolerance/ignorance to melanoma differentiation antigens and enhances antitumor immunity. J Immunol 2006, 176(11):6434-6442.

25. Hu P, Arias RS, Sadun RE, Nien YC, Zhang N, Sabzevari H, Lutsiak ME, Khawli LA, Epstein AL: Construction and preclinical characterization of Fc-mGITRL for the immunotherapy of cancer. Clin Cancer Res 2008, 14(2):579-588.

26. Shimizu J, Yamazaki S, Takahashi T, Ishida Y, Sakaguchi S: Stimulation of CD25(+)CD4(+) regulatory T cells through GITR breaks immunological self-tolerance. Nat Immunol 2002, 3(2):135-142.

27. Stephens GL, McHugh RS, Whitters MJ, Young DA, Luxenberg D, Carreno BM, Collins M, Shevach EM: Engagement of glucocorticoid-induced TNFR family-related receptor on effector $T$ cells by its ligand mediates resistance to suppression by CD4 + CD25+ T cells. J Immunol 2004, 173(8):5008-5020.

28. Yao $S, Z$ hu $Y$, Chen L: Advances in targeting cell surface signalling molecules for immune modulation. Nat Rev Drug Discov 2013, 12(2):130-146

29. Dai M, Wei H, Yip YY, Feng Q, He K, Popov V, Hellstrom I, Hellstrom KE: Long-lasting complete regression of established mouse tumors by counteracting Th2 inflammation. J Immunother 2013, 36(4):248-257.

30. Guo Z, Cheng D, Xia Z, Luan M, Wu L, Wang G, Zhang S: Combined TIM-3 blockade and CD137 activation affords the long-term protection in a murine model of ovarian cancer. J Transl Med 2013, 11(1):215.

31. Cohen AD, Schaer DA, Liu C, Li Y, Hirschhorn-Cymmerman D, Kim SC, Diab A, Rizzuto G, Duan F, Perales MA, et al: Agonist anti-GITR monoclonal antibody induces melanoma tumor immunity in mice by altering regulatory T cell stability and intra-tumor accumulation. PLoS One 2010, 5(5):e10436.

32. Ko K, Yamazaki S, Nakamura K, Nishioka T, Hirota K, Yamaguchi T, Shimizu J, Nomura T, Chiba T, Sakaguchi S: Treatment of advanced tumors with agonistic anti-GITR mAb and its effects on tumor-infiltrating Foxp3 + CD25 + CD4+ regulatory T cells. J Exp Med 2005, 202(7):885-891.

33. Quezada SA, Peggs KS, Curran MA, Allison JP: CTLA4 blockade and GM-CSF combination immunotherapy alters the intratumor balance of effector and regulatory T cells. J Clin Invest 2006, 116(7):1935-1945.

34. Curran MA, Kim M, Montalvo W, Al-Shamkhani A, Allison JP: Combination CTLA-4 blockade and 4-1BB activation enhances tumor rejection by increasing T-cell infiltration, proliferation, and cytokine production. PLoS One 2011, 6(4):e19499.

35. Johnston FM, Tan MC, Tan BR Jr, Porembka MR, Brunt EM, Linehan DC, Simon PO Jr, Plambeck-Suess S, Eberlein TJ, Hellstrom KE, et al: Circulating mesothelin protein and cellular antimesothelin immunity in patients with pancreatic cancer. Clin Cancer Res 2009, 15(21):6511-6518.

36. Ho M, Hassan R, Zhang J, Wang QC, Onda M, Bera T, Pastan I: Humoral immune response to mesothelin in mesothelioma and ovarian cancer patients. Clin Cancer Res 2005, 11(10):3814-3820.

37. Cho JS, Hsu JV, Morrison SL: Localized expression of GITR-L in the tumor microenvironment promotes CD8+ T cell dependent anti-tumor immunity. Cancer Immunol Immunother 2009, 58(7):1057-1069.
38. Wei H, Hellstrom KE, Hellstrom I: Elafin selectively regulates the sensitivity of ovarian cancer cells to genotoxic drug-induced apoptosis. Gynecol Oncol 2012, 125(3):727-733.

39. Kim YH, Choi BK, Kim KH, Kang SW, Kwon BS: Combination therapy with cisplatin and anti-4-1BB: synergistic anticancer effects and amelioration of cisplatin-induced nephrotoxicity. Cancer Res 2008, 68(18):7264-7269.

40. Zitvogel L, Galluzzi L, Smyth MJ, Kroemer G: Mechanism of action of conventional and targeted anticancer therapies: reinstating immunosurveillance. Immunity 2013, 39(1):74-88.

\section{doi:10.1186/1479-5876-12-36}

Cite this article as: Lu et al.: Combined PD-1 blockade and GITR triggering induce a potent antitumor immunity in murine cancer models and synergizes with chemotherapeutic drugs. Journal of Translational Medicine 2014 12:36.

\section{Submit your next manuscript to BioMed Central and take full advantage of:}

- Convenient online submission

- Thorough peer review

- No space constraints or color figure charges

- Immediate publication on acceptance

- Inclusion in PubMed, CAS, Scopus and Google Scholar

- Research which is freely available for redistribution 